\title{
Novel Approach for Simulations of in vivo Mechanical Stimulations - A Perspective for Mechanotherapy
}

\section{Tzyy Yue Wong ${ }^{1}$, Pei-Wen Cheng ${ }^{1}$, Rong-Chang Jhong ${ }^{1}$, Chia-Ti Tsai Tsai ${ }^{2}$, Jou-Wei Lin ${ }^{3}$, Juey- Jen Hwang ${ }^{2}$, Sheng-Nan Chang ${ }^{3,4}$}

${ }^{I}$ Department of Medical Education and Research, Kaohsiung Veterans General Hospital, Kaohsiung, Taiwan, ${ }^{2}$ Division of Cardiology, Department of Internal Medicine, National Taiwan University Hospital, Taipei, Taiwan, ${ }^{3}$ Division of Cardiology, Department of Internal Medicine, National Taiwan University Hospital Yun-Lin Branch, Dou-Liu City, Taiwan, ${ }^{4}$ National Taiwan University College of Medicine, Graduate Institute of Clinical Medicine, Taipei, Taiwan

\section{Background}

Physical forces are important for maintaining cellular functions. Recently, mechanotherapy, a field that deals with mechanical stimulation connections with Biological functions at cellular and molecular levels has become the emerging hot topic in clinical medicine. Many mammalian cell types contain mechanosensor protein, from cells such as ear, nasal, skin epithelium, colon, muscle, kidney, to lung cells. The forces experienced by living cells such as cyclic (heartbeat), sustained (skin), cyclic and sustained stretching (rectal muscle) can be simulated by in vitro approach. Since evidence on mechanical stimulation-induced Biological functions is still lacking, we will study the various forces based on their physiological functions and meanings. We hypothesize that stretching of cells by in vitro approach can regulate cell functions that have physiological meaning.

Methods

A company based in Taiwan has developed a device with four modules for stretching. First, we will examine the stretching modules using 1) normal (skin epithelial cells) and 2) pathological cells (lung, skin, colon, or oral cancer cells) to stretch cells to study the cytoskeletal structure actin expression using immunofluorescence assay, and collagen secretion by Sirius Red staining. Second, we will develop 3D culture system using gelatin scaffold in which we will focus on cell adhesion (Ecadherin expression), proliferation (BrdU: 5-bromo-2'-deoxyuridine incorporation), and the secreted extracellular matrix (Collagen staining).

Results

We tested lung cancer cells (A549), skin epithelial cancer cells (A431), colon cancer cells (WiDr), and oral cancer cells (SCC-25) for adhesion to the membrane coated with 1) non-coated, 2) collagen-coated, 3) Nitrogen Hydroxide (NH2)coated surfaces that are stretchable. The A549 and A431 cells were subjected to cyclic stretching (5\%, 1Hz, 6h). Results showed that actin filaments were organized in different pattern compared to that of No stretch. The study on the correlation between stretching and cellular function at molecular level is ongoing.

Conclusions

The effect of cyclic stretching on the various cell types show differences in actin distribution which may pose specific Biological function. As a result, we will continue to establish the criteria for mimicking the in vivo using the mechanical stimulation device.

Keywords

Mechanotherapy, physical forces, cyclic and sustained stretching 